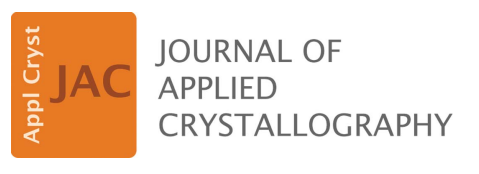

ISSN 1600-5767

Received 6 June 2017

Accepted 4 October 2017

Edited by G. Kostorz, ETH Zurich, Switzerland

Keywords: phenol red dye; crystal growth; FT-Raman spectroscopy; scanning electron microscopy; SEM; optical properties; dielectric response; photoluminescence.

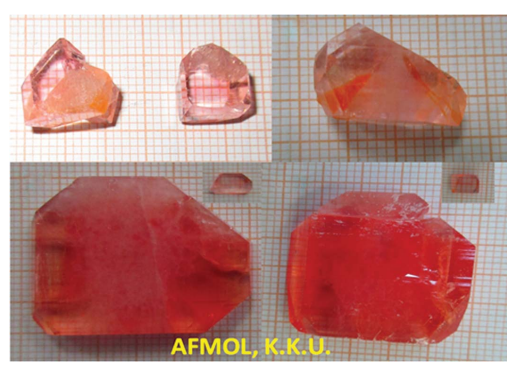

C 2017 International Union of Crystallography

\section{Effect of phenol red dye on monocrystal growth, crystalline perfection, and optical and dielectric properties of zinc (tris) thiourea sulfate}

\author{
Mohd. Shkir, ${ }^{a *}$ V. Ganesh, ${ }^{\text {a }}$ S. AlFaify, ${ }^{a *}$ K. K. Maurya ${ }^{b}$ and N. Vijayan ${ }^{b}$ \\ advanced Functional Materials and Optoelectronic Laboratory (AFMOL), Department of Physics, King Khalid University, \\ PO Box 9004, Abha, 61413, Saudi Arabia, and ${ }^{\mathbf{b}}$ National Physical Laboratory, Council of Scientific and Industrial \\ Research, Dr K. S. Krishnan Road, New Delhi, 110012, India. *Correspondence e-mail: \\ shkirphysics@gmail.com,shkirphysics@kku.edu.sa,sasaalfaify@hotmail.com
}

In this work, the growth of large size $(\sim 25 \times 29 \times 5 \mathrm{~mm}$ and $\sim 25 \times 24 \times 6 \mathrm{~mm})$ colorful single crystals of zinc (tris) thiourea sulfate (ZTS) in the presence of 0.05-2 wt\% phenol red (PR) dye was achieved using a simple and low-cost technique. Powder X-ray diffraction patterns confirm the presence of PR dye, which is indicated by an enhancement of the Raman peak intensities, a shift in their position and the appearance of a few extra peaks. The quality of the grown crystals was assessed by high-resolution X-ray diffraction, which shows that the crystalline perfection of $1 \mathrm{wt} \%$ PR-dyed ZTS crystals is better than that of $2 \mathrm{wt} \%$ PR-dyed crystals. The measured UV-vis absorbance spectra show two additional, strong absorption bands at $\sim 430$ and $558 \mathrm{~nm}$ in the dyed crystals, due to the presence of PR dye, along with a band at $\sim 276 \mathrm{~nm}$ which is present for all crystals but is slightly shifted for the dyed crystals. Photoluminescence spectra were recorded at two excitation wavelengths $\left(\lambda_{\mathrm{exc}}=310\right.$ and $\left.385 \mathrm{~nm}\right)$. The luminescence intensity is found to be enriched in dyed crystals, with some extra emission bands. An enhancement in the value of the dielectric constant and a.c. electrical conductivity was also observed in the dyed ZTS crystals.

\section{Introduction}

Recently, nonlinear optical (NLO) materials have been found to have important applications in, for example, opto-electronic, photonic, data conversion, retrieval, storage and frequency-doubling devices (Saleh \& Teich, 1991; Penn et al., 1991; Shakir et al., 2009; Shakir, Kushwaha et al., 2010; Shkir, Abbas et al., 2014; Shkir, AlFaify et al., 2015; Badan et al., 1993; Zaitseva \& Carman, 2001; Shkir, Muhammad, AlFaify et al., 2015; Shkir, Muhammad \& AlFaify, 2015). Luminescent dyed crystals exhibit better properties than polymers and glasses as they possess superior thermal conductivity, low dispersion and intrinsic polarization, have fewer defects, and can be used in laser devices (Yang \& Ozin, 2000, Wanke et al., 1997). Benedict et al. (2003) studied dying processes in crystals. A review on dyeing of different crystal faces has also been published recently (Kahr \& Shtukenberg, 2016; Kahr \& Gurney, 2001).

Zinc (tris) thiourea sulfate (ZTS) single crystals have good NLO properties compared to standard NLO materials such as potassium dihydrogen phosphate (Dhumane et al., 2008). Having such a key characteristic, ZTS is a valuable component in high-energy lasers as a frequency convertor. Recently, the growth of dyed ZTS crystals has been reported; the grown crystals had modified physical properties that make them suitable for linear, nonlinear and piezoelectric applications (Bhandari et al., 2014; Shkir, 2016; Shkir et al., 2016). These 
results show that the properties of ZTS crystals are strongly affected when they are grown in the presence of dye or with dye doping.

Phenol red (PR) is well known as a $\mathrm{pH}$ indicator, usually applied in cell culture, and has a wide range of applications (Berthois et al., 1986; Mills \& Skinner, 2011). According to the current available literature, there have been no reports on ZTS crystal growth in the presence of PR dye so far. In the present work, our aim is to grow good quality colorful single crystals of ZTS with different concentrations of PR dye, which we denote as PRZTS. The $\mathrm{pH}$ value of all the prepared solutions for crystal growth was measured. Furthermore, the grown crystals were subjected to X-ray diffraction and FTRaman analyses to confirm structural and vibrational changes, respectively, high-resolution X-ray diffraction (HRXRD) to assess the crystalline perfection, UV-vis spectroscopy for band gap analysis, photoluminescence (PL) spectroscopy to study the fluorescence behavior, and dielectric and mechanical studies to discern the defects and strength of the grown crystals.

\section{Experimental}

\subsection{Synthesis and crystal growth}

High-purity zinc sulfate heptahydrate $\left(\mathrm{ZnSO}_{4} \cdot 7 \mathrm{H}_{2} \mathrm{O}\right)$, thiourea $\left[\mathrm{CS}\left(\mathrm{NH}_{2}\right)_{2}\right]$ and PR dye were purchased from SigmaAldrich. $\mathrm{ZnSO}_{4} \cdot 7 \mathrm{H}_{2} \mathrm{O}$ and $\mathrm{CS}\left(\mathrm{NH}_{2}\right)_{2}$ in a 1:3 ratio were dissolved in two different beakers containing double distilled (DD) water. Pure ZTS $\left\{\mathrm{Zn}\left[\mathrm{CS}\left(\mathrm{NH}_{2}\right)_{2}\right]_{3} \cdot \mathrm{SO}_{4}\right\}$ was synthesized by mixing the two solutions in another beaker using a temperature-controlled magnetic stirrer (Isotemp, Fisher Scientific) above room temperature (i.e. $323 \pm 1 \mathrm{~K}$ ). The chemical reaction between these two species to obtain the final product is

$$
\mathrm{ZnSO}_{4} \cdot 7 \mathrm{H}_{2} \mathrm{O}+3 \mathrm{CS}\left(\mathrm{NH}_{2}\right)_{2} \rightarrow \mathrm{Zn}\left[\mathrm{CS}\left(\mathrm{NH}_{2}\right)_{2}\right]_{3} \cdot \mathrm{SO}_{4} .
$$

A similar procedure was followed to prepare another four sets of ZTS solution, to which were added different concen- trations of PR dye, from 0.05 to $2 \mathrm{wt} \%$ (i.e. from 0.0036 to $0.146 \mathrm{~g})$. The solutions were again mixed thoroughly by regular stirring $\left(700 \mathrm{r} \mathrm{min}^{-1}\right)$ for $24 \mathrm{~h}$ at the same temperature and red solutions were obtained as a result of the dye. The $\mathrm{pH}$ of all the prepared solutions was measured using a $\mathrm{pH}$ meter, and the variation of $\mathrm{pH}$ with $\mathrm{PR}$ dye content is shown in Fig. 1(a). It can be concluded that the $\mathrm{pH}$ is strongly affected by the addition of dye to the parent solution.

All the prepared solutions were left at the same temperature to evaporate the solvent and obtain the salts of the pure and dyed ZTS which were further used for single-crystal growth. Again, the synthesized materials were dissolved in DD water at the same temperature with the aim of growing good quality single crystals. The temperature was reduced to $300 \pm 1 \mathrm{~K}$ in steps of $1 \mathrm{~K}$ per $12 \mathrm{~h}$ and the solution was carefully watched. It was found that on reducing the temperature nucleates were formed, and these were dissolved by adding an appropriate amount of DD water. Finally the prepared saturated solutions were filtered using filter paper into new beakers. All of the beakers were then covered with a pierced lid and kept in a constant temperature bath at $300 \pm$ $1 \mathrm{~K}$. The solutions were observed frequently to avoid any multiple nucleations. Fig. 1 shows examples of the grown crystals of pure ZTS [see the inset of Fig. 1(a)] and ZTS with $0.05,0.1,1$ and $2 \mathrm{wt} \%$ PR dye addition, respectively (Fig. $1 b$, iiv). It is clear that the pure crystal is transparent white; however, in the presence of dye the crystal becomes colored. The crystals shown in Fig. 1 were harvested from their respective solutions after about $60 \mathrm{~d}$. The size of the grown crystals with $1 \mathrm{wt} \%$ dye is $\sim 25 \times 29 \times 5 \mathrm{~mm}$ and with $2 \mathrm{wt} \%$ dye is $\sim 2 \times 24 \times 6 \mathrm{~mm}$. From the figure it is also clear that the dye is homogeneously adsorbed in the ZTS crystals at higher concentrations and the morphology is greatly modified. From the insets of Figs. 1(a) and 1(b), it is clear that the faces such as (100), (001), (101), (011), (011) and (010) present in the pure ZTS crystal (Fig. 1a) are suppressed as a result of the presence of the dye and growth is taking place along the [110] direction. For further studies, we have chosen the crystals grown at higher dye concentrations (i.e. 1 and $2 \mathrm{wt} \%$ ) along with the pure crystal.

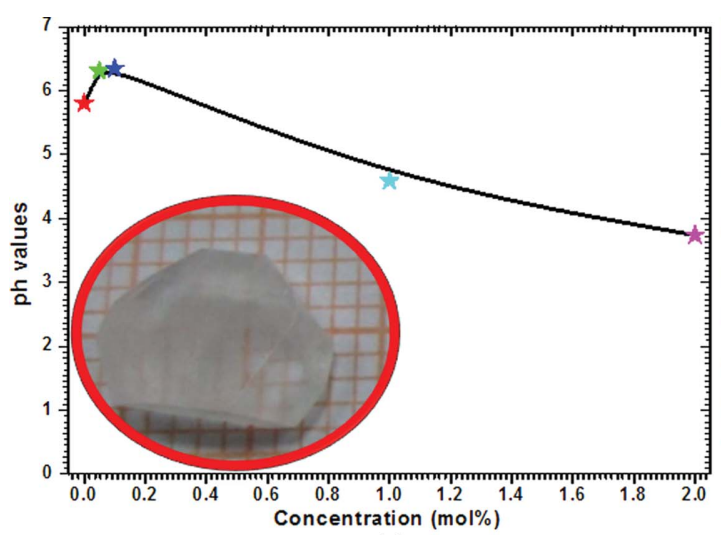

(a)

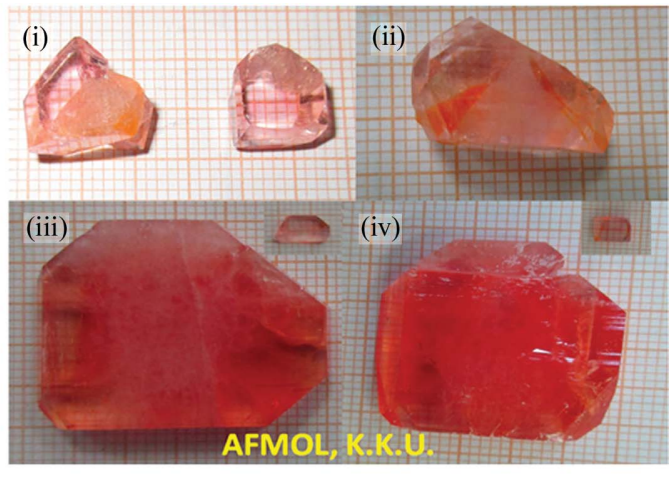

(b)

Figure 1

(a) Variation of $\mathrm{pH}$ values and (b) as-grown single crystals of ZTS with (i) $0.05 \mathrm{wt} \%$, (ii) $0.10 \mathrm{wt} \%$, (iii) $1 \mathrm{wt} \%$ and (iv) $2 \mathrm{wt} \% \mathrm{PR}$ dye. 


\subsection{Characterization methods}

Some of the grown ZTS and PRZTS crystals were crushed to fine powders for $\sim 5 \mathrm{~min}$ and used to record powder X-ray diffraction patterns using a Shimadzu (Japan) XRD-6000 powder X-ray diffractometer with $\mathrm{Cu} K \alpha$ radiation, $\lambda=$ $0.1543 \mathrm{~nm}$, operated at $40 \mathrm{kV}, 30 \mathrm{~mA}$ at a scan rate of $0.2^{\circ} \mathrm{m}^{-1}$ over an angular range of $5-70^{\circ}$ at $300 \mathrm{~K}$. As-grown crystals [shown in the insets of Figs. 1( $a)$ and 1(b) were used to record the FT-Raman spectra on a Thermo Scientific DXR FTRaman spectrometer in the wavenumber range of 3500$50 \mathrm{~cm}^{-1}$. The crystalline perfection was assessed by HRXRD (PANalytical X'Pert PRO MRD system) using $\mathrm{Cu} K \alpha_{1}$ radiation ( $\lambda=1.540598 \AA$ ). Scanning electron microscopy (SEM) (JSM 6360 LA, JEOL Ltd, Tokyo, Japan) was used to record the surface topography. UV-vis absorbance spectra were measured using a JASCO 570 UV-vis-NIR spectrophotometer in the wavelength range from 190 to $1000 \mathrm{~nm}$. A Thermo Fisher Scientific Lumina fluorescence spectrometer was used to record the PL spectra in the wavelength range of $380-700 \mathrm{~nm}$ at $300 \mathrm{~K}$. The dielectric and a.c. electrical conductivity measurement was done in a high frequency range using a Keithley 4200-SCS system at $300 \mathrm{~K}$.

\section{Results and discussion}

\subsection{Structural studies}

Fig. 2(a) shows measured X-ray diffraction patterns for the pure PR dye, pure ZTS and dyed ZTS powdered specimens. It is clear from the figure that the intensity of the diffraction peaks has been increased because of the dye, which indicates the good crystalline quality of the dyed crystals. On comparing the diffraction patterns of the pure dye and the pure and dyed ZTS crystals it is observed that there are some extra peaks in the patterns of the dyed crystals along with some shifting in the peaks' positions (see Fig. $2 b$ ). The extra peaks observed at $13.08^{\circ}(021), 18.49^{\circ}(212), 21.25^{\circ}(321), 24.54^{\circ}(322)$ and $31.24^{\circ}$ (432) are due to the presence of the PR dye (JCPDS No. 1224041 ) in the ZTS crystals (see Fig. $2 b$ ). The intensity of the 211, 406 and 617 peaks is found to be particularly enhanced for
Table 1

Refined lattice parameters of pure and dyed ZTS crystals.

\begin{tabular}{|c|c|c|c|c|}
\hline \multirow[b]{2}{*}{$\begin{array}{l}\text { Lattice } \\
\text { parameters }\end{array}$} & \multirow{2}{*}{$\frac{\text { Previous work } \dagger}{\text { ZTS }}$} & \multicolumn{3}{|c|}{ Current work $\neq$} \\
\hline & & ZTS & PRZTS (1 wt \%) & PRZTS (2 wt \%) \\
\hline$a(\AA)$ & 11.0673 & 11.0607 & 11.0812 & 11.0809 \\
\hline$b(\AA)$ & 7.7342 & 7.7331 & 7.7366 & 7.7516 \\
\hline$c(\AA \circ)$ & 15.5573 & 15.5741 & 15.5521 & 15.5454 \\
\hline$V\left(\AA^{3}\right)$ & 1331.65 & 1332.10 & 1333.29 & 1335.264 \\
\hline
\end{tabular}

$\dagger$ Cole \& Hickstein (2013). $\$$ Refined by CHECKCELL.

$2 \mathrm{wt} \%$ PR-dyed ZTS crystals. For the confirmation of the crystal system and lattice parameter calculations, the recorded XRD data were used for refinement in the CHECKCELL software (Laugier \& Bochu, 2000). The peak positions were determined by basic data processing using the XRD-6000 software (Shimadzu, Kyoto, Japan) and high-intensity peaks were used as input in the above-mentioned software to obtain accurate refined parameters. The number of peaks is found to be increased in the dyed crystals. The refinement confirms that the grown crystals have orthorhombic crystal structure with space and point groups of $P c a 2_{1}$ and $m m 2$, respectively. The refined lattice parameters are presented in Table 1 and are found to be in close agreement with previously reported values (Andreetti et al., 1968; Ushasree, Jayavel \& Ramasamy, 1999; Ushasree, Jayavel, Subramanian \& Ramasamy, 1999; Moitra \& Kar, 2007; Cole \& Hickstein 2013). It can be seen from Table 1 that the lattice parameters vary with PR concentration.

\subsection{Vibrational (FT-Raman and FT-IR spectroscopy) studies}

The measured FT-Raman spectra of the ZTS and PRZTS crystals in the characteristic wavenumber range are shown in Fig. 3(a). It is seen that the Raman intensity and transparency are significantly increased in the ZTS crystals grown in the presence of dye. This indicates an enhancement in fluorescence activity of PRZTS crystals. The FT-Raman spectrum for the higher wavenumber range is not shown here; however, all

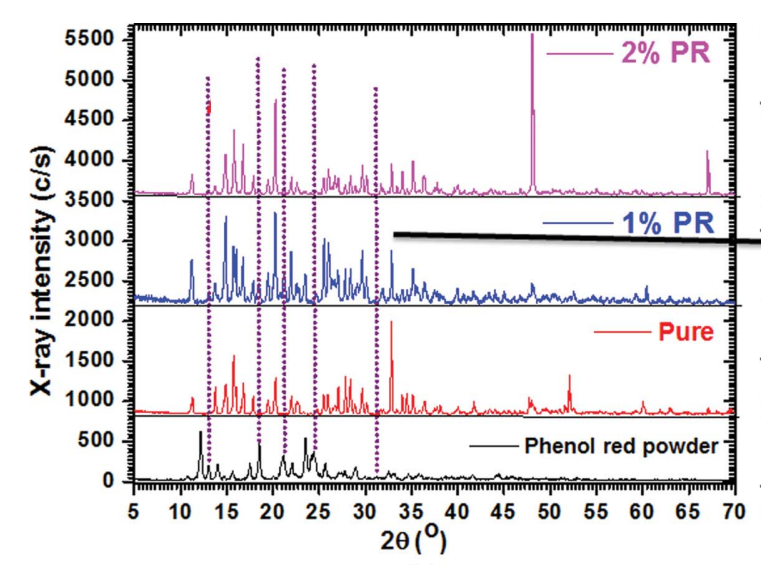

(a)

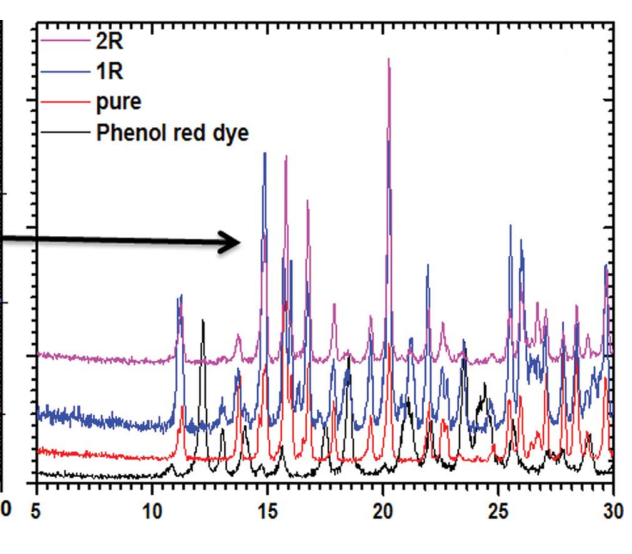

(b)

Figure 2

(a) Powder X-ray diffraction patterns and (b) close-up view in the angular range of 5-30 . 
Table 2

Observed vibrational modes (FT-Raman) and their corresponding assignments.

\begin{tabular}{llll}
\hline \multicolumn{2}{l}{ Observed wavenumbers $\left(\mathrm{cm}^{-1}\right)$} & & \\
\cline { 1 - 2 } ZTS & PRZTS $(1 \mathrm{wt} \%)$ & PRZTS $(2 \mathrm{wt} \%)$ & Assignments \\
\hline $96,66,50,35,24$ & $92,64,45,37$ & $95,65,47,33,23$ & Lattice vibration \\
$152,125,110$ & $150,123,111$ & $151,124,110$ & Lattice vibration \\
238 & 234 & 235 & Lattice vibration \\
265 & 262 & 261 & $\mathrm{Zn}-\mathrm{S}$ \\
430 & 431 & 429 & $v\left(\mathrm{SO}_{4}\right)$ \\
485 & 480 & 483 & Vibration $(\mathrm{Zn}-\mathrm{S})$, \\
& & & $(\mathrm{Zn}-\mathrm{O})$ \\
530 & 514,533 & 525,546 & $v(\mathrm{Zn}-\mathrm{O})$ \\
592,602 & 605 & 595 & $\left.v(\mathrm{SO})_{4}\right)$ \\
715 & 711 & 713 & $v(\mathrm{C}-\mathrm{N}), v_{\mathrm{s}}(\mathrm{C}=\mathrm{S})$ \\
953 & 955 & 951 & $v\left(\mathrm{SO} \mathrm{S}_{4}\right)$ \\
1027 & 1037 & 1026 & $\mathrm{~S}=\mathrm{C}-\mathrm{N}$ \\
1090 & 1088 & 1089 & $\rho\left(\mathrm{NH}_{2}\right)$ \\
1109 & 1108 & 1111 & $v\left(\mathrm{SO}_{4}\right)$ \\
1408 & 1395 & 1404 & $v_{\text {as }}(\mathrm{CS})$ \\
1438 & 1435 & 1440 & $v_{\text {as }}(\mathrm{CS}), v_{\text {as }}(\mathrm{CN})$ \\
1513 & 1514 & 1512 & $v_{\text {as }}(\mathrm{CN}), \mathrm{S}=\mathrm{C}-\mathrm{N}$, \\
& & & $\mathrm{N}-\mathrm{C}-\mathrm{N}$ \\
1633,1651 & 1643,1661 & 1645,1667 & $\delta\left(\mathrm{NH}_{2}\right)$ \\
3213 & 3211 & 3208 & $v_{\mathrm{s}}\left(\mathrm{NH}_{2}\right)$ \\
3351 & 3342,3390 & 3340 & $v_{\text {as }}\left(\mathrm{NH}_{2}\right)$ \\
\hline
\end{tabular}

the bands observed in this range can be assigned to symmetric and asymmetric vibrations of $\mathrm{NH}$ stretching of several $\mathrm{N}-$ $\mathrm{H}$...O bonds in the ZTS with minute shifts due to the presence of the dye. The vibrational modes for the ZTS and PRZTS crystals are presented in Table 2. Raman modes at $\sim 1513,1438$ and $1408 \mathrm{~cm}^{-1}$ in ZTS occur because of stretching and asymmetric stretching vibrations of $\mathrm{N}-\mathrm{C}-\mathrm{N}$ and $\mathrm{C}=\mathrm{S}$ bonds, respectively (Venkataramanan et al., 1994). However, these bands are found to be shifted in the dyed crystals (see Table 2). The vibration bands at 1408 and $715 \mathrm{~cm}^{-1}$ arise from metal-sulfur bonding of zinc with thiourea. The positions of these bands in the dyed crystals are also found to be shifted, as can be seen from Table 2. These bands are in good correlation with earlier reports (Venkataramanan et al., 1994). The bands due to stretching and rocking vibrations of $\mathrm{SO}_{4}$ and $\mathrm{NH}_{2}$ are seen at $\sim 1109,953,602,430$ and $1090 \mathrm{~cm}^{-1}$, respectively, in
ZTS, but these bands are clearly shifted in the dyed crystals. The Raman band at $1027 \mathrm{~cm}^{-1}$ is due to $\mathrm{S}=\mathrm{C}-\mathrm{N}$ vibrations. Raman bands due to symmetric stretching and rocking vibrations of $\mathrm{C}=\mathrm{S}$ and $\mathrm{C}-\mathrm{N}$ mode occur at $\sim 715$ and $485 \mathrm{~cm}^{-1}$ in ZTS and show a clear shift in position for PRZTS crystals (see Table 2). The band due to stretching vibration of the $\mathrm{Zn}-\mathrm{S}$ bond is seen at $265 \mathrm{~cm}^{-1}$ in ZTS, but is also shifted in the PRZTS crystals (Venkataramanan et al., 1994). The other vibrational modes listed in Table 2 are due to lattice vibrations in ZTS, and these are also seen in PRZTS with a shift in their positions. The shift in peak positions shows that there is a clear interaction between the dye and ZTS, which is also confirmed by the color of the crystals (see Fig. 1) and the XRD study. The bands due to the presence of dye are not quite detectable by the Raman laser instrument owing to the low concentration of the dye, but they may be identified by surface-enhanced FT-Raman spectroscopy.

Furthermore, we have recorded FT-IR spectra for ZTS and PRZTS to see the effect of the dye on the vibrational modes, as shown in Fig. 3(b). It can be clearly seen in the figure that all the grown crystals have similar vibrational frequencies (Bhandari et al., 2014; Shkir, 2016; Selvapandiyan et al., 2013). However, some new bands, as well as band shifts, were observed in the dyed crystals. The strong band due to plane bending of the $\mathrm{NH}_{2}$ group which occurs at $1632 \mathrm{~cm}^{-1}$ in ZTS is found to be shifted to 1628 and $1627 \mathrm{~cm}^{-1}$ in 1 and $2 \mathrm{wt} \%$ PRZTS crystals. The band due to stretching vibration of the $\mathrm{N}-\mathrm{C}-\mathrm{N}$ bond which is observed at $1517 \mathrm{~cm}^{-1}$ in ZTS is found to be shifted to 1511 and $1507 \mathrm{~cm}^{-1}$ in the PRZTS crystals. The band due to stretching vibration of $\mathrm{SO}_{4}$ which occurs at $1128 \mathrm{~cm}^{-1}$ in ZTS is shifted to 1127 and $1107 \mathrm{~cm}^{-1}$ in the respective PRZTS crystals. The band observed at $1090 \mathrm{~cm}^{-1}$ in ZTS becomes more broad and seems to disappear in the dyed crystals. The band observed at $1031 \mathrm{~cm}^{-1}$ in ZTS is shifted to 1030 and $1021 \mathrm{~cm}^{-1}$ in the respective dyed crystals. The band due to $\mathrm{SO}_{4}$ at $590 \mathrm{~cm}^{-1}$ in ZTS is observed to be at 581 and $577 \mathrm{~cm}^{-1}$ in the respective dyed crystals. A very broad low-intensity vibrational band at $1470 \mathrm{~cm}^{-1}$ was observed, and this may be assigned to stretching vibration of

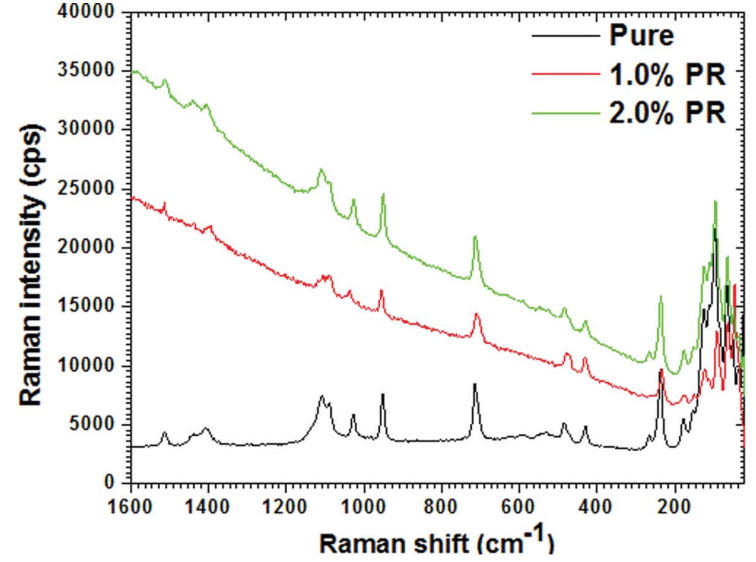

(a)

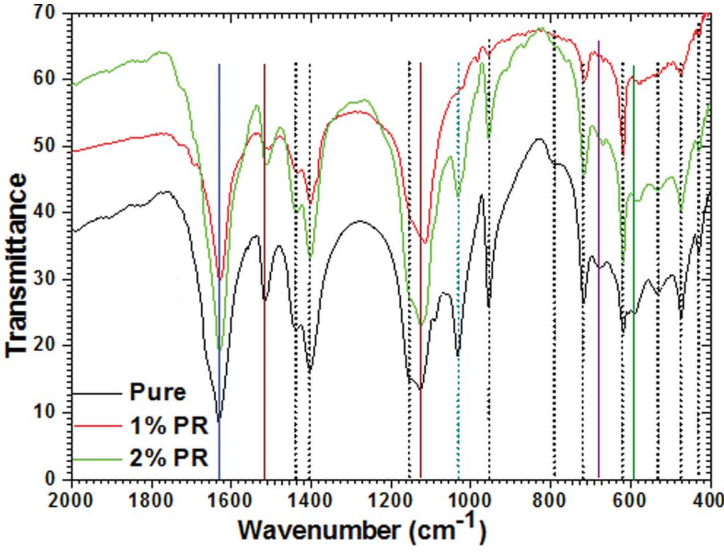

(b)

Figure 3

(a) FT-Raman and (b) FT-IR spectra of ZTS and PRZTS crystals 
the sulfonate group of the PR dye (which is seen at $\sim 1460 \mathrm{~cm}^{-1}$ in the pure dye) (Wahab \& Hussain, 2016). This band is not easily visible in the current spectra. However, by zooming in on the specific range of the spectra it can be found. A new broad and sharp vibrational band observed at $982 \mathrm{~cm}^{-1}$ in the PRZTS crystals may be due to the PR dye. However, this band has been reported in pure $P R$ at $1016 \mathrm{~cm}^{-1}$ (Wahab \& Hussain, 2016). The other two new bands at 866 and $908 \mathrm{~cm}^{-1}$ that are observed in the dyed crystals may also be due to the PR dye, as these bands in the pure dye are reported at $862 / 840$ and $919 / 912 \mathrm{~cm}^{-1}$ (Wahab \& Hussain, 2016). The occurrence of these bands provides clear evidence of the dye in the ZTS crystals.

\subsection{Surface topography study by SEM}

Capturing the surface topography using SEM can help us to assess the quality of the grown crystals to some extent. SEM images for as-grown single crystals and their surface topographs at lower and higher resolution are shown in Fig. 4. It can be seen that the surface morphology of ZTS has been modified by the PR dye compared to the pure crystals reported in our previous studies (Shkir, 2016). The SEM images clearly indicate that the dye has been adsorbed on the surface of the ZTS crystals. The surface of the $2 \mathrm{wt} \%$ PR-dyed crystal is clearly affected compared to the $1 \mathrm{wt} \%$ PR-dyed crystal. The $2 \mathrm{wt} \%$-dyed crystal surface contains etch-pit-like structures on the surface when we test the surface at low scale, which are not present in the $1 \mathrm{wt} \%$-dyed crystals at the same scale. This shows that the surfaces of the ZTS crystals grown in the presence of higher dye concentrations are more affected than those grown at low concentrations.

\subsection{High-resolution X-ray diffraction studies}

The HRXRD diffraction curves for the (200) diffraction planes of pure and 1 and $2 \mathrm{wt} \%$ PR-dyed ZTS crystals are shown in Fig. 5. The curve in Fig. 5(a) is quite sharp and possess a single peak with a very low full width at halfmaximum (FWHM) of the order of $8^{\prime \prime}$, which is quite close to that expected for an ideally perfect single crystal according to the dynamical theory of X-ray diffraction (Batterman \& Cole, 1964; Shakir, Kushawaha et al., 2010). The sharp nature of the intensity versus glancing angle curve shows that this crystal contains a very low density of point defects and their agglomerates. On very close observation of the curve, there is a slight asymmetry between the negative and positive sides with respect to the exact Bragg peak position (comparison curve is not shown), which indicates that the ZTS crystal predominantly contains vacancy-type defects. The same has been reported previously (Kushwaha et al., 2011). However, the value of FWHM reported here for the pure ZTS crystal is slightly less than the earlier reported value (Kushwaha et al., 2011). The low value of FWHM indicates the better crystalline perfection of the single crystals reported in the current work, which directly indicates better growth conditions. Fig. 5(b) shows the diffraction curve of the $1 \mathrm{wt} \%$ PR-dyed ZTS crystal, which is broader than that of the pure ZTS crystal. The presence of PR has yielded a significant increase in the FWHM value, from 8 to $19^{\prime \prime}$, and clear asymmetry caused by increased intensity on the negative side. The asymmetry is clearly visible since the broadening of the overall diffraction curve has been increased owing to the inclusion of the PR dye in the ZTS lattice. This diffraction curve clearly shows the presence of predominantly vacancy defects in the $1 \mathrm{wt} \% \mathrm{PR}$ dyed ZTS crystal. The higher concentration of $2 \mathrm{wt} \%$ PR dye leads to a more symmetric and broader diffraction curve, having an FWHM value of $84^{\prime \prime}$ (Fig. 5a). Although this curve looks very symmetric in nature, towards the positive side the scattering intensity is much higher compared to the negative side, which is due to the presence of a grain boundary. The grain boundary is separated by $\sim 763^{\prime \prime}$ from the main peak (see Fig. 5c). The appearance of this grain boundary at $2 \mathrm{wt} \% \mathrm{PR}$
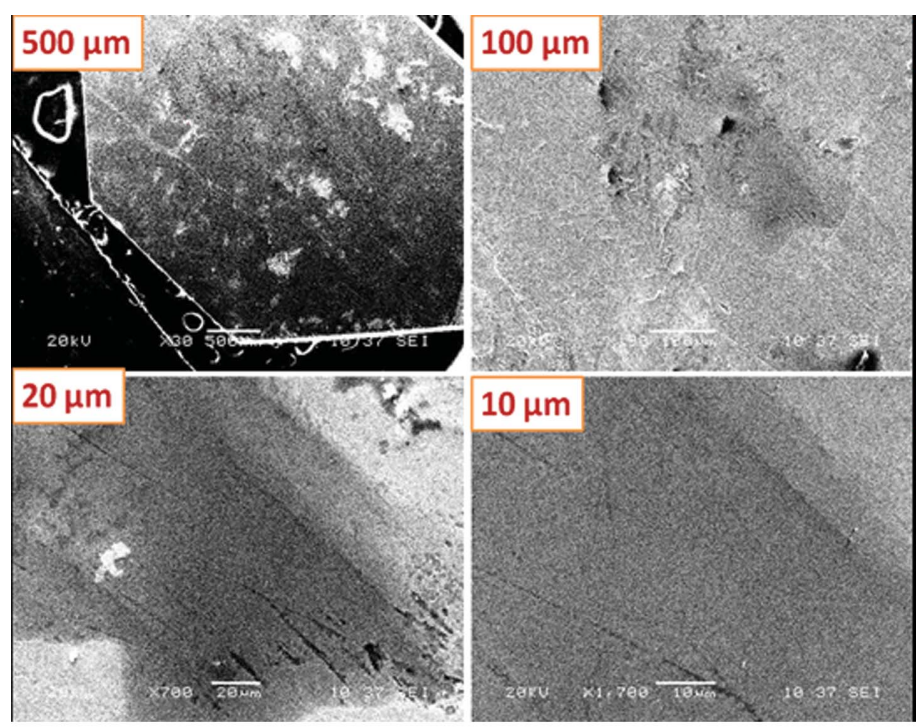

(a)

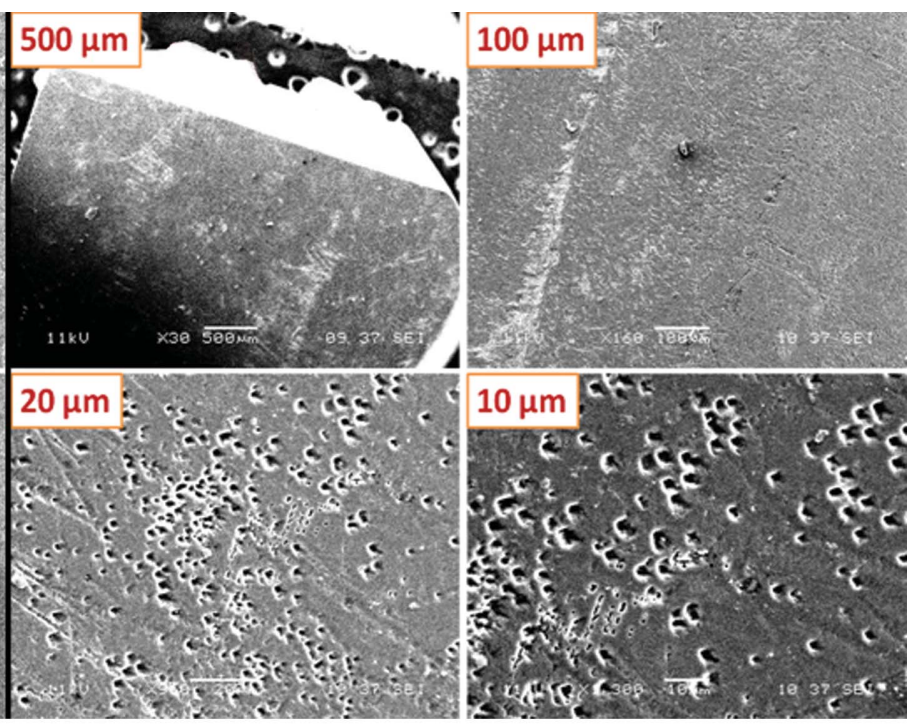

(b)

Figure 4

SEM topographs of (a) $1 \mathrm{wt} \%$ and (b) $2 \mathrm{wt} \%$ PRZTS single crystals. 

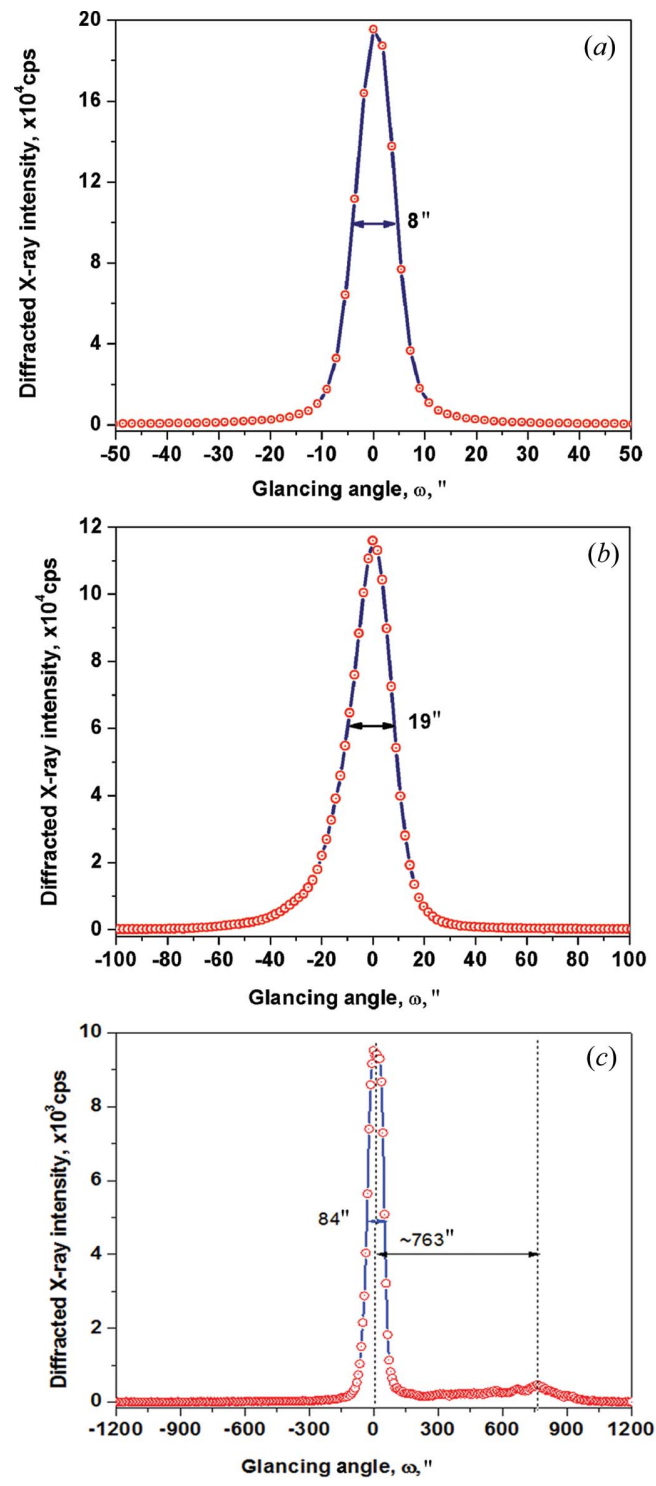

Figure 5

HRXRD diffraction curves for $(a)$ pure, $(b) 1 \mathrm{wt} \%$ PR-dyed and (c) 2 wt \% PR-dyed ZTS single crystals. dye concentration indicates the upper limit of possible interaction of PR dye in ZTS crystals.

\subsection{UV-visible spectroscopy analysis}

To establish the effect of the dye on the grown crystals' optical applications, we measured absorbance spectra from colloidal solutions of the crystals in double distilled water, as shown in Fig. 6(a). It can be seen from the figure that all the grown crystals of ZTS show much less absorbance in the wavelength range of 300-1000 nm. However, the dyed crystals have peaks in their spectra in the wavelength range of 600-1000 nm. This indicates that the grown crystals can be used in particular wavelength ranges in optoelectronic devices. In the pure and 1 and $2 \mathrm{wt} \%$-dyed ZTS crystals absorption bands are observed at 276, 274 and $272 \mathrm{~nm}$, respectively. However, two more absorption bands with strong absorption values are observed at $430 \pm 2$ and $558 \pm 2 \mathrm{~nm}$ in both PRZTS crystals. These bands are due to the presence of the PR dye and are shifted in comparison to the spectrum of the pure PR dye (Rovati et al., 2012). These bands clearly show that the dye has a very strong effect on the ZTS crystalline matrix.

Furthermore, the optical band gap was calculated from the Tauc plot for the ZTS and PRZTS crystals. For energy gap calculation first we calculated the absorption coefficient $\alpha$ using the well known Beer-Lambert law relation, $\alpha=2.303 A / R$, where $A$ is the $\mathrm{UV}$-vis absorbance and $R$ is the path length of the quartz cuvette $(10 \mathrm{~mm})$ used during the measurement. The Tauc plots for PRZTS crystals are shown in Fig. 6(b). The value of $\alpha$ for the pure ZTS crystal is found to be $\sim 0.02$. However, in the dyed crystals this value is significantly increased and it is found to be $\sim 0.14$ and 0.17 at $\sim 194 \mathrm{~nm}$ wavelength. To evaluate the energy gap we have extrapolated a straight line from the $(\alpha h v)^{2}$ versus $h v$ curve to the point of intersection with the $x(h v)$ axis (Fig. 6b). The value of the energy gap is found to be 4.32, 4.29 and $4.25 \mathrm{eV}$ for ZTS and 1 and $2 \mathrm{wt} \%$-dyed PRZTS crystals, respectively. The energy gap value is found to be reduced in the dyed crystals compared to the pure crystal, which is a clear indication of dye interaction

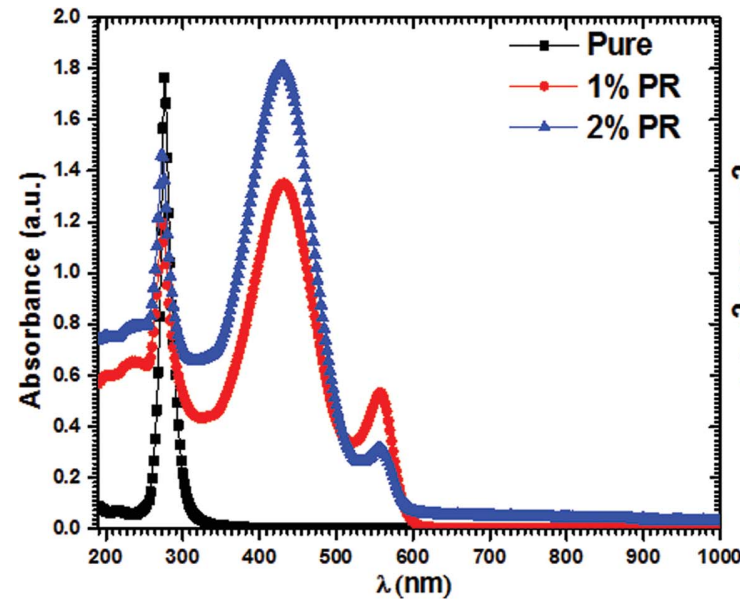

(a)

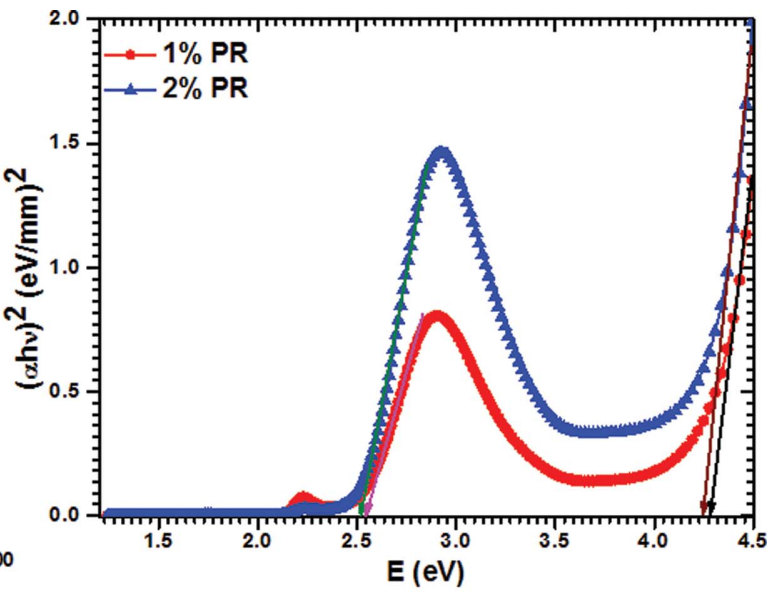

(b)

Figure 6

(a) Absorbance spectra and (b) energy gap plots for ZTS and PRZTS crystals. 
with the ZTS matrix. Such a reduction in energy gap has also been calculated from diffuse reflectance (DR) data for powdered specimens of dyed ZTS crystals (Shkir et al., 2016; Shkir, 2016). However, the value of the energy gap is found to be lower compared to our previous reports in which these values were calculated using DR data by the Kubelka-Munk method (Shkir et al., 2016; Shkir, 2016). The band gap values for pure and dyed ZTS crystals have also been reported to be 4.16-4.18, 4.046, 4.1818-4.1995 and 4.54 eV (Muley, 2014; Rao \& Kalainathan, 2012; Selvapandiyan et al., 2013; Bhandari et al., 2014). The difference in band gap of pure ZTS may be due to a change in cutoff wavelength, and that may also depend on the quality of the crystals and lattice variation. Because there are two more absorption bands in the PRZTS crystals due to the PR dye, two more band gaps were computed. These were found to be $\sim 2.15$ and $2.5 \mathrm{eV}$ in the two dyed crystals. Owing to the high band gap, the grown crystals may be applied in electro-optic devices (Periyasamy et al., 2007; Shkir, Riscob et al., 2014).

\subsection{Photoluminescence analysis}

Figs. 7(a) and 7(b) show the measured PL emission spectra for the ZTS and PRZTS single crystals at $300 \mathrm{~K}$. The two excitation wavelengths $\lambda_{\text {exc }}=310$ and $385 \mathrm{~nm}$ were used to record the emission spectra of each of the crystals. The $310 \mathrm{~nm}$ PL spectra of ZTS and 1 and $2 \mathrm{wt} \%$ PR-dyed ZTS crystals have a UV emission band at $\sim 368,361$ and $361 \mathrm{~nm}$, respectively, with enhanced PL intensity and a slight shift in peak position for the dyed crystals. A violet-blue emission band is also observed at $430 \mathrm{~nm}$ in the pure crystals, which becomes very broad in the dyed crystals and seems to disappear. There is a new broad green emission band at $\sim 520 \mathrm{~nm}$ in the dyed crystals.

However, when the grown crystals were excited at $\sim 385 \mathrm{~nm}$ then a violet-blue emission band at $447 \mathrm{~nm}$ was observed for pure and dyed crystals, as shown in Fig. 6(b). The PL intensity of this band was enhanced with increasing dye content. The emission bands at $\sim 430$ in pure and 447 in dyed crystals may be due to $\mathrm{S}^{2-}$ vacancies (Bhandari et al., 2014; Kushwaha et al., 2011, 2014; Rao \& Kalainathan, 2012) in the ZTS crystals.

Two more emission bands are also observed, at $\sim 525 \mathrm{~nm}$ (intense) and $575 \mathrm{~nm}$ (broad), in the dyed crystals. These extra bands may be due to interaction of the PR dye with ZTS. Such a band for pure PR dye is reported at $545 \mathrm{~nm}$ when excited at $350 \mathrm{~nm}$ (Zarei \& Ghazanchayi, 2016). The intense emission band at $\sim 578 \mathrm{~nm}$ was also reported in phenol red as fluorophore in a poly(vinyl alcohol) membrane matrix when excited at $386 \mathrm{~nm}$ (Zarei \& Ghazanchayi, 2016). The bands are shifted to some extent and several new bands occurred in dyed ZTS crystals. These PL results suggest that the PR dye is strongly interacting with the ZTS matrix.

\subsection{Dielectric and a.c. electrical conductivity analyses}

For dielectric studies, the capacitance $(C)$, impedance $(Z)$ and loss tangent $(\tan \delta)$ were measured in the frequency range from $3 \mathrm{kHz}$ to $10 \mathrm{MHz}$. The dielectric constant $\left(\varepsilon_{1}\right)$ and loss $\left(\varepsilon_{2}\right)$ were evaluated using the well known relations given below (Kaygili et al., 2013, 2015):

$$
\begin{gathered}
\varepsilon_{1}=\frac{C d}{\varepsilon_{0} A}, \\
\varepsilon_{2}=\varepsilon_{1} \tan \delta,
\end{gathered}
$$

where $\varepsilon_{0}$ is the permittivity of free space $\left(\varepsilon_{0}=8.854 \times\right.$ $\left.10^{-12} \mathrm{~F} \mathrm{~m}^{-1}\right), d$ and $A$ are the thickness and area of the crystal sample.

Fig. 8(a) shows a plot of the variation of the relative permittivity $\left(\varepsilon_{1}\right)$ values as a function of frequency for all the ZTS and 1 and $2 \mathrm{wt} \%$-dyed PRZTS crystals. It is apparent that $\varepsilon_{1}$ is dependent on frequency in all of the crystals. The value of $\varepsilon_{1}$ is found to be almost stable in the whole tested frequency range, as we have performed this measurement in the higher frequency range. It can also be seen from Fig. $8(a)$ that $\varepsilon_{1}$ is increased in the dyed crystals from $\sim 12$ to $\sim 26$, which is higher than the previously reported value (Bhandari et al.,

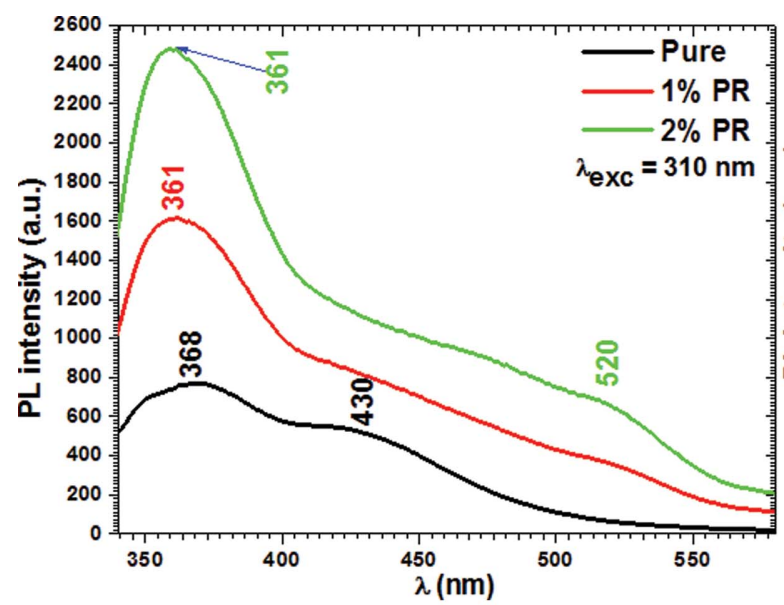

(a)

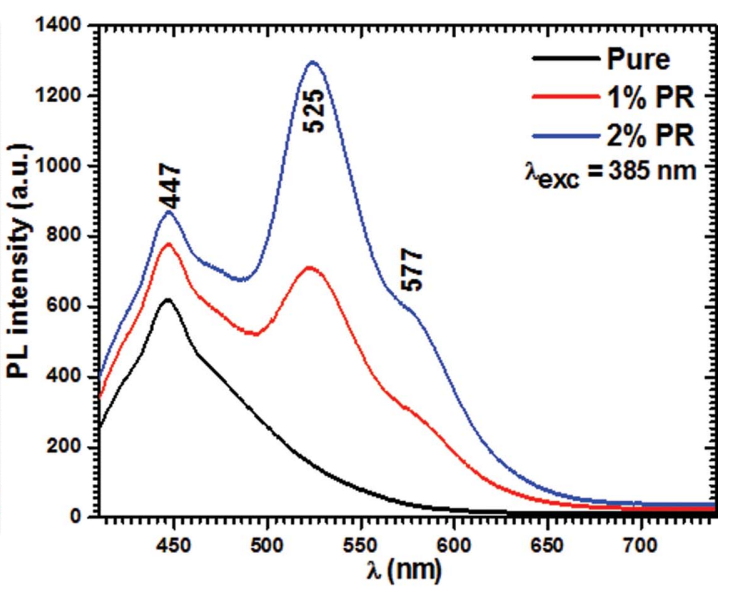

(b)

Figure 7

PL emission spectra for grown crystals excited at (a) $310 \mathrm{~nm}$ and (b) $385 \mathrm{~nm}$. 


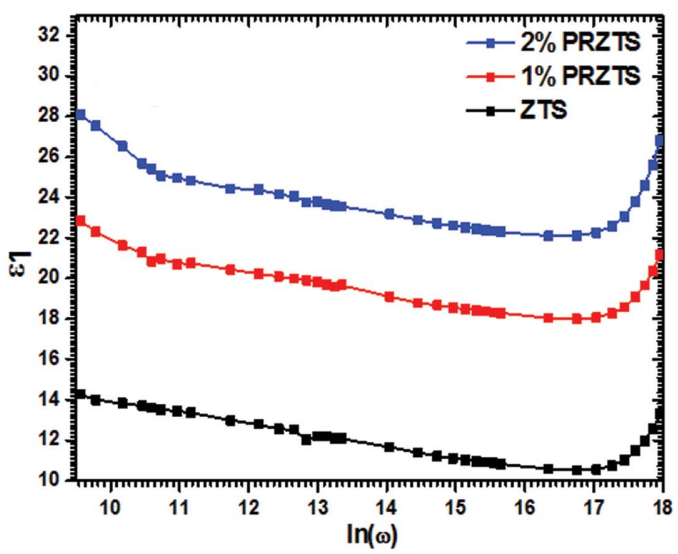

(a)

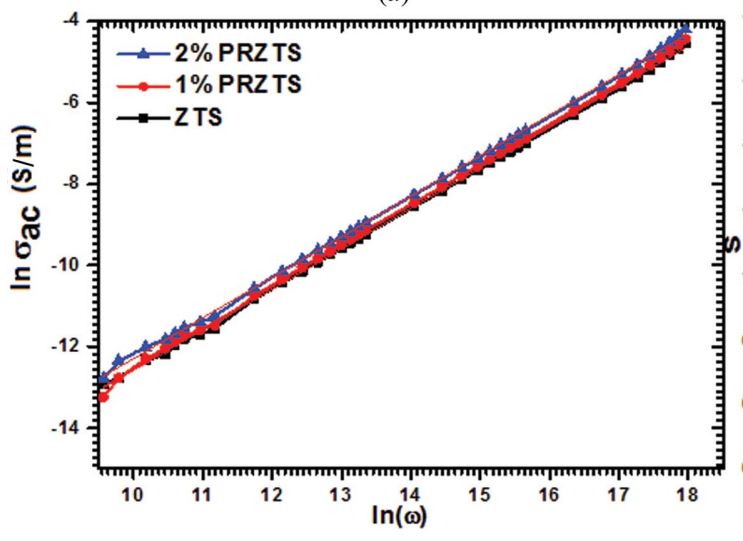

(c)

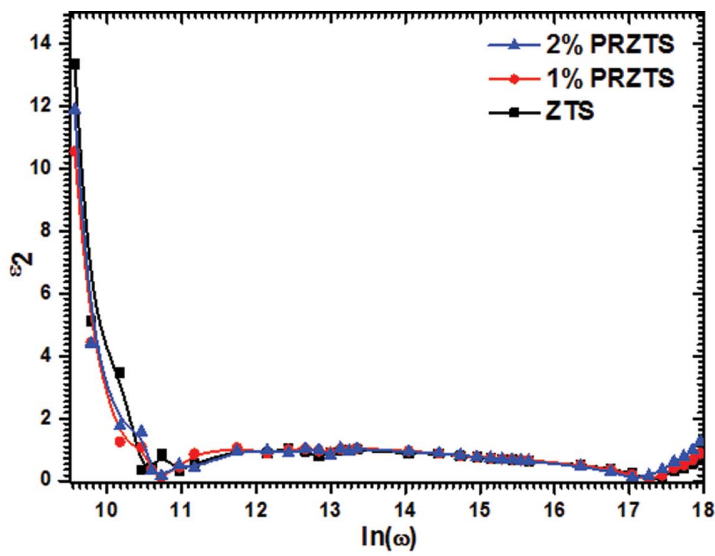

(b)

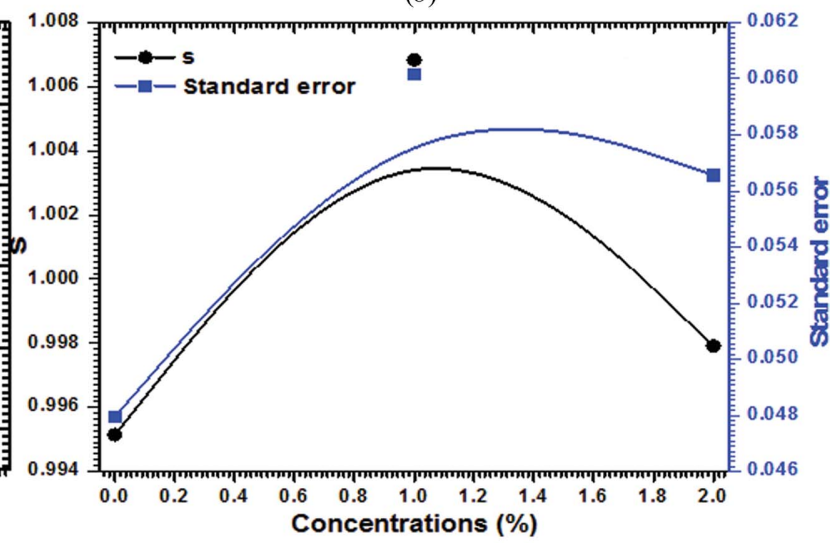

(d)

Figure 8

Plots of variation of $(a) \varepsilon_{1},(b) \varepsilon_{2},(c) \ln \sigma_{\text {ac }}$ and $(d) s$ for ZTS and PRZTS crystals.

2014). Such enhancement in dyed crystals may be due to the high dielectric polarization of the added dye. The $\varepsilon_{2}$ values show similar behavior to $\varepsilon_{1}$, as shown in Fig. $8(b)$, and the low values confirm that the grown crystals contain few defects.

Furthermore, the total a.c. electrical conductivity $\left(\sigma_{\text {tot.ac }}\right)$ value was calculated using the following relations (Kaygili et al., 2013, 2015):

$$
\begin{gathered}
\sigma_{\text {tot.ac }}=\frac{d}{Z A}, \\
\sigma_{\text {tot.ac }}=\sigma_{\text {dc }}+B \omega^{s} .
\end{gathered}
$$

Here, $\sigma_{\mathrm{dc}}$ is the direct current conductivity, $B$ is a constant, $\omega$ is the angular frequency and $s$ is the frequency exponent. Fig. 8(c) shows a plot of the variation of the total a.c. electrical conductivity with frequency. An increase in the value of $\ln \sigma_{\mathrm{ac}}$ is observed with increasing frequency in the grown crystals, following the universal power law. The frequency exponent $(s)$ value was also determined from the slope of the linear part of the $\ln \sigma_{\text {ac }}$ versus $\ln \omega$ curve (Fig. $8 c$ ) and found to be between 0.99514 and 1.00684 , as shown in Fig. $8(d)$. The value of $s$ is almost equal to unity for all the tested crystals. As per the available literature the value of $s$ for ionic conducting materials is between 0.6 and 1 , but its theoretical limit is 1 (Lee et $a l ., 1991)$. The calculated value of $s$ is found to be $\leq 1$, which shows that the hopping mechanism of conduction in the studied material involves a translational motion with sudden carrier hopping within the grown crystals.

\section{Conclusion}

Large-size dyed single crystals of zinc (tris) thiourea sulfate have been grown in the presence of different concentrations of phenol red dye using a simple solution method at $300 \mathrm{~K}$. The size of the grown crystals with $1 \mathrm{wt} \%$ dye is $\sim 25 \times 29 \times 5 \mathrm{~mm}$ and with $2 \mathrm{wt} \%$ dye is $\sim 25 \times 24 \times 6 \mathrm{~mm}$. These crystals were grown in about 60 days. The presence of the dye was proved by a robust structural and vibrational analysis. The lattice parameters are found to be affected in the presence of the dye, but the phase was not affected. The degree of crystalline perfection of pure and PR-dyed ZTS single crystals was assessed using HRXRD and it was found that the grown single crystals have very good crystalline perfection and few defects or grain boundaries. Concentrations of $<2 \mathrm{wt} \%$ PR dye in ZTS may yield crystals with no grain boundary, since the $2 \mathrm{wt} \%$ PR dye concentration has given some indication of developing grain boundaries, as shown in Fig. 5(c). The surface morphology was studied by SEM and found to be strongly affected by the presence of the dye. The visible change in color throughout the crystals shows that the dye is homogeneously present in ZTS crystals at higher concentrations. In pure and 1 and $2 \mathrm{wt} \%$-dyed ZTS crystals a strong absorption band is observed 
at 276, 274 and $272 \mathrm{~nm}$, respectively. However, two more absorption bands with strong absorption values are observed at $430 \pm 2$ and $558 \pm 2 \mathrm{~nm}$ in both PRZTS crystals. The value of the energy gap is found to be $4.32,4.29$ and $4.25 \mathrm{eV}$ for the ZTS and PRZTS crystals, respectively. Owing to the presence of more absorption bands in ZTS crystals grown in the presence of PR dye, two more band gaps were also computed and these were found to be $\sim 2.15$ and $2.5 \mathrm{eV}$. The PL spectra of the ZTS and PRZTS single crystals excited at $310 \mathrm{~nm}$ show a UV emission band at $\sim 368,361$ and $361 \mathrm{~nm}$, respectively, with enhanced PL intensity for the dyed crystals. However, under excitation at $\sim 385 \mathrm{~nm}$, a violet-blue emission band at $\sim 447 \pm 2 \mathrm{~nm}$ with increasingly enhanced PL intensity was observed in all crystals. The enhancement of PL intensity shows the formation of defects which act as color centers. The value of the dielectric constant for ZTS crystals is improved when they are grown in the presence of dye. The mechanical strength of the crystals was also found to be improved. The enhanced properties of dyed crystals suggest that they may have broad applications in the field of linear and nonlinear optical devices.

\section{Funding information}

The authors would like to express their gratitude to the Deanship of Scientific Research, King Khalid University, Saudi Arabia, for providing financial support under project No. R.G.P. 2/3/38.

\section{References}

Andreetti, G. D., Cavalca, L. \& Musatti, A. (1968). Acta Cryst. B24, 683-690.

Badan, J., Hierle, R., Perigaud, A., Zyss, J. \& Williams, D. (1993). NLO Properties of Organic Molecules and Polymeric Materials, American Chemical Society Symposium Series. Washington, DC: American Chemical Society.

Batterman, B. W. \& Cole, H. (1964). Rev. Mod. Phys. 36, 681-717.

Benedict, J. B., Wallace, P. M., Reid, P. J., Jang, S. H. \& Kahr, B. (2003). Adv. Mater. 15, 1068-1070.

Berthois, Y., Katzenellenbogen, J. A. \& Katzenellenbogen, B. S. (1986). Proc. Natl Acad. Sci. USA, 83, 2496-2500.

Bhandari, S., Sinha, N., Ray, G. \& Kumar, B. (2014). Chem. Phys. Lett. 591, 10-15.

Cole, J. M. \& Hickstein, D. D. (2013). Phys. Rev. B, 88, 184105.

Dhumane, N. R., Hussaini, S. S., Dongre, V. G. \& Shirsat, M. D. (2008). Opt. Mater. 31, 328-332.

Kahr, B. \& Gurney, R. W. (2001). Chem. Rev. 101, 893-951.

Kahr, B. \& Shtukenberg, A. (2016). CrystEngComm, 18, 8988-8998.

Kaygili, O., Dorozhkin, S. V., Ates, T., Canan Gursoy, N., Keser, S., Yakuphanoglu, F. \& Birkan Selçuk, A. (2015). Mater. Sci. Eng. C, 47, 333-338.

Kaygili, O., Keser, S., Ates, T., Al-Ghamdi, A. A. \& Yakuphanoglu, F. (2013). Powder Technol. 245, 1-6.
Kushwaha, S. K., Maurya, K. K., Haranath, D. \& Bhagavannarayana, G. (2011). J. Appl. Cryst. 44, 1054-1061.

Kushwaha, S., Maurya, K., Vijayan, N., Gupta, A., Haranath, D., Kumar, B., Kanjilal, D. \& Bhagavannarayana, G. (2014). Nucl. Instrum. Methods Phys. Res. Sect. B, 338, 1-7.

Laugier, J. \& Bochu, B. (2000). CHECKCELL. Laboratoire des Materiaux et du Génie Physique de l'Ecole Supérieure de Physique de Grenoble, France.

Lee, W., Liu, J. F. \& Nowick, A. (1991). Phys. Rev. Lett. 67, 1559-1561.

Mills, A. \& Skinner, G. A. (2011). Analyst, 136, 894-896.

Moitra, S. \& Kar, T. (2007). Opt. Mater. 30, 508-512.

Muley, G. (2014). J. Elec Mater. 43, 439-446.

Penn, B. G., Cardelino, B. H., Moore, C. E., Shields, A. W. \& Frazier, D. (1991). Prog. Cryst. Growth Charact. Mater. 22, 19-51.

Periyasamy, B. K., Jebas, R. S. \& Thailampillai, B. (2007). Mater. Lett. 61, 1489-1491.

Rao, R. H. \& Kalainathan, S. (2012). Spectrochim. Acta A Mol. Biomol. Spectrosc. 97, 456-463.

Rovati, L., Fabbri, P., Ferrari, L. \& Pilati, F. (2012). Fiber Optic Sensors, edited by M. Yasin, ch. 17. InTech. https://doi.org/10.5772/ 26517.

Saleh, B. E. A. \& Teich, M. C. (1991). Fundamentals of Photonics, Wiley Series in Pure and Applied Optics, pp. 644-695. New York: John Wiley and Sons.

Selvapandiyan, M., Arumugam, J., Sundaramoorthi, P. \& Sudhakar, S. (2013). J. Alloys Compd. 558, 34-38.

Shakir, M., Kushawaha, S. K., Maurya, K. K., Kumar, S., Wahab, M. A. \& Bhagavannarayana, G. (2010). J. Appl. Cryst. 43, 491-497.

Shakir, M., Kushwaha, S., Maurya, K., Bhatt, R., Rashmi, Wahab, M. A. \& Bhagavannarayana, G. (2010). Mater. Chem. Phys. 120, 566-570.

Shakir, M., Singh, B., Kumar, B. \& Bhagavannarayana, G. (2009). Appl. Phys. Lett. 95, 252902.

Shkir, M. (2016). J. Mater. Res. 31, 1046-1055.

Shkir, M., Abbas, H., Kumar, S., Bhagavannarayana, G. \& AlFaify, S. (2014). J. Phys. Chem. Solids, 75, 959-965.

Shkir, M., AlFaify, S., Abbas, H. \& Bhagavannarayana, G. (2015). Mater. Chem. Phys. 155, 36-46.

Shkir, M., AlFaify, S., Ganesh, V., Yahia, I., Algarni, H. \& Shoukry, H. (2016). J. Mater. Sci. Mater. Electron. 27, 10673-10683.

Shkir, M., Muhammad, S. \& AlFaify, S. (2015). Spectrochim. Acta A Mol. Biomol. Spectrosc. 143, 128-135.

Shkir, M., Muhammad, S., AlFaify, S., Irfan, A. \& Yahia, I. (2015). Spectrochim. Acta A Mol. Biomol. Spectrosc. 137, 432-441.

Shkir, M., Riscob, B., Hasmuddin, M., Singh, P., Ganesh, V., Wahab, M., Dieguez, E. \& Bhagavannarayana, G. (2014). Opt. Mater. 36, 675-681.

Ushasree, P. M., Jayavel, R. \& Ramasamy, P. (1999). Mater. Sci. Eng. $B, \mathbf{6 5}, 153-158$.

Ushasree, P., Jayavel, R., Subramanian, C. \& Ramasamy, P. (1999). J. Cryst. Growth, 197, 216-220.

Venkataramanan, V., Srinivasan, M. \& Bhat, H. (1994). J. Raman Spectrosc. 25, 805-811.

Wahab, H. S. \& Hussain, A. A. (2016). J. Nanostruct. Chem. 6, 261274.

Wanke, M. C., Lehmann, O., Müller, K., Wen, Q. \& Stuke, M. (1997). Science, 275, 1284-1286.

Yang, S. M. \& Ozin, G. A. (2000). Chem. Commun. pp. 2507-2508.

Zaitseva, N. \& Carman, L. (2001). Prog. Cryst. Growth Charact. Mater. 43, 1-118.

Zarei, A. R. \& Ghazanchayi, B. (2016). Talanta, 150, 162-168. 